\title{
VPDP: A Service Discovery Protocol for Ubiquitous Computing
}

\author{
Zhaomin Xu, Ming Cai, and Jinxiang Dong \\ Institute of Artificial Intelligence, Zhejiang University, 310027 Hangzhou, China \\ $\{x z m, c m, d j x\} @ z j u . e d u . c n$
}

\begin{abstract}
Many service discovery protocols have been proposed by far to support ubiquitous computing. But they don't always apply to ubiquitous computing because of their confines. In this paper, we propose a service discovery protocol named VPDP adopted in our DOM-based middleware architecture, which aims to support as many types of ubiquitous environments as possible. VPDP is important for our middleware architecture to accommodate heterogeneity and uncertainty of ubiquitous computing environments.
\end{abstract}

Keywords: Ubiquitous computing, Service discovery, Volunteer, Middleware.

\section{Introduction}

The essence of the vision about ubiquitous computing was the creation of environments saturated with computing and communication capability, yet gracefully integrated with human users [1]. The technological advances necessary to build a ubiquitous computing environment fall into four broad areas: devices, networking, middleware, and applications [2].

Service discovery is essential for ubiquitous computing environments to gracefully integrate networked computing devices. Service discovery protocols are designed to minimize administrative overhead and increase usability. They can also save ubiquitous system designers from trying to foresee and code all possible interactions and states among devices and programs at design time. [3]. In this paper, we propose a service discovery protocol called VPDP (Ubiquitous Discovery Protocol based on Volunteers) for ubiquitous computing, which aims to support as many types of ubiquitous environments as possible. This protocol can accommodate heterogeneity and uncertainty of ubiquitous computing environments. Volunteers in our middleware architecture are middleware nodes with less limited resources.

The rest of this paper is structured as follows: Section 2 discusses our service discovery protocol including volunteers election, service registration and service discovery. Section 3 shows the experimental result of service discovery using VPDP. Finally, we make a conclusion of this paper and describe our future work. 


\section{Ubiquitous Discovery Protocol Based on Volunteers}

\subsection{Volunteers Election}

Volunteers in VSD [5] are elected within a one-hop network range using broadcasts. In our middleware architecture, volunteers are elected from middleware nodes within the range of a certain number of hops using multicasts. The number of hops (denoted as 'TTLs') is defined as a system parameter which can be changed by system administrators or by the middleware system automatically. The election method of volunteers in our middleware architecture is similar to VSD, but we have made a few modifications to its parameters. Fig. 1 shows the node state transition diagram of VPDP.

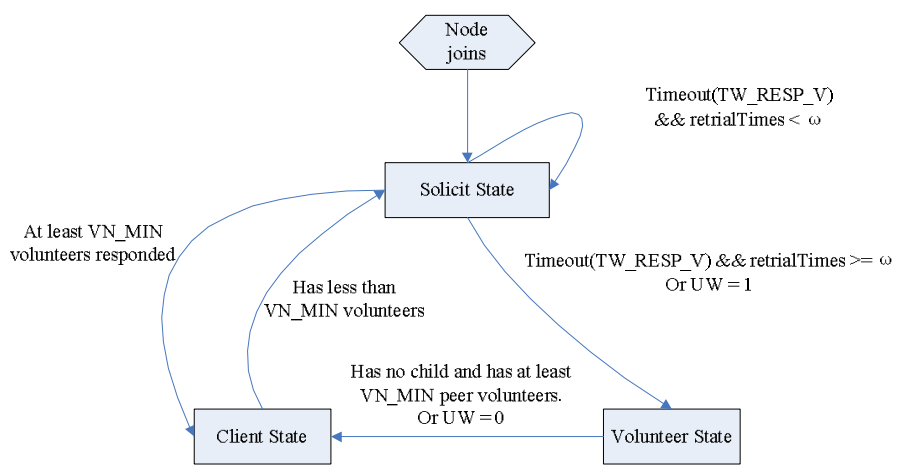

Fig.1. Node state transition diagram

Unlike VSD, in our middleware architecture, when a node repeats the solicit process after it can't register with k volunteers within a given time period, it will try to register with $\max \left\{(\mathrm{k} / 2), \mathrm{k}_{\min }\right\}$ volunteers. Although at the start all nodes try to register with $\mathrm{k}$ volunteers, they may end up with different number of local volunteers that they have registered with. In other words, each client may have different number of local volunteers and each volunteer may maintain different number of the same directory entries for different clients.

In VSD, each node sets its own retrial times (denoted as ' $\omega$ ') by considering its willingness, degree of mobility and amount of resource [5]. The lower value of $\omega$ the higher chance a node can take to be a volunteer. In our middleware architecture, each node turns on or restarts with the system parameter $\omega$ set to a default value, which will be changed by a evaluation function over time.

The evaluation function of $\omega$ depends on three parameters: the total running time in hours (TRTh) of the node since it turns on or restarts, changed times of the network address (CTNA) of the node, and the number of services $\left(\mathrm{n}_{\mathrm{s}}\right)$ on the node. The evaluation function is invoked periodically or when some events happen (such as network address changed, new services mounted on the node). TRTh and CTNA indicate the degree of mobility of a node. The number of services indicates the 
amount of resources on the node. The willingness of a node is hard to evaluate. We use the system parameter user willingness (UW, a value between 0 and 1 , high value indicates high willingness) and $n_{\mathrm{s}}$ to evaluate a node's willingness. Currently, the evaluation function used in our middleware architecture is defined as below.

$$
\omega=\frac{1}{U W \times\left(n_{s}+1\right)} \times \frac{C T N A+1}{T R T h / 7} \times \frac{6}{n_{s}+1}
$$

\subsection{Service Registration and Discovery}

When a SP registers its services, it sends service advertisements to its local volunteers one by one through unicasts. A SP will register its services with at least $\mathrm{k}_{\min }$ volunteers, which form a logical overlay network. Volunteers extract service information from service advertisements received and store services' information in their service cache. Volunteers' announcements contain advertisements about their cached services, so that service advertisements can spread over the network.

A service requestor (SR) can send its service request messages if it has at least $\mathrm{k}_{\min }$ local volunteers. There are two types of queries as stated in paper [4] in our middleware architecture: one query-one response (1/1) and one query-multiple responses $(1 / \mathrm{n})$. In $1 / \mathrm{n}$ queries, the service discovery process is almost the same as that described in paper [5].

In $1 / 1$ queries, the SR selects a volunteer in its local volunteers list and sends a service request to it. If the SR gets a service response, it can then directly interact with the SP. Otherwise if it can't get any response within a certain amount of time, it will send the service request to the next volunteer in its local volunteers list. If all the volunteers have been tried and the SR still can't get any service response, the discovery process fails. On receiving a service request, a volunteer will lookup services in its service cache. The volunteer will send the service request to the first matched SP. If the SP accepts the request, it will send an acknowledgement to both the SP and the volunteer. The service discovery process ends successfully. Else if the SP doesn't respond within a certain amount of time, the volunteer will try to find another matched SP in its service directory. If the volunteer can't find any matched $\mathrm{SP}$, it will forward the service request to its nearby volunteers. The discovery process will continue in this way until a matched SP is found or TTLr $<=0$ (the time to live field of the request message) or all neighbor volunteers have been tried.

\section{Experiment}

We have developed a prototype of our DOM-based middleware architecture using Eclipse 3 and J2SDK 1.4.2. The experiment is taken in our campus network which is composed of many Local Area Networks. We have tested the mean response time of a service request within different number of nodes. Fig. 2 shows the results which indicate that VPDP is very efficient in service discovery. 


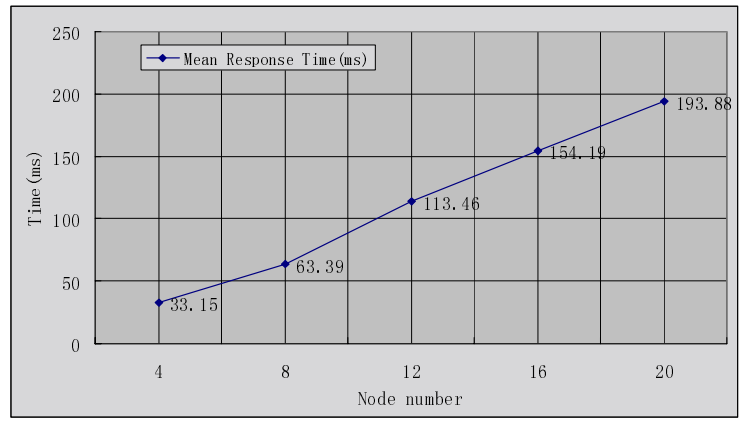

Fig. 2. Results of service discovery using VPDP

\section{Conclusions and Future Work}

Service discovery is essential for ubiquitous computing environments to gracefully integrate networked computing devices. We have discussed the VPDP protocol adopted in our middleware architecture, which aims to support as many types of ubiquitous environments as possible. Experimental results show that VPDP is efficient in service discovery, which is important for our middleware architecture to accommodate heterogeneity and uncertainty of ubiquitous computing environments. In the future, we will keep on improving VPDP to realize efficient service migration.

\section{References}

1. M., Satyanarayanan: Pervasive Computing: Vision and Challenges. IEEE Personal Communications, 8(4) (2001) 10-17

2. D., Saha, A., Mukherjee: Pervasive computing: a paradigm for the 21 st century. Computer, 36(3) (2003) 25-31

3. Feng, Zhu, Matt, W., Mutka, Lionel, M., Ni: Service discovery in pervasive computing environments. IEEE Pervasive Computing, 4(4) (2005) 81-90

4. Celeste, Campo, Carlos, García-Rubio, Andrés, Marín López, Florina, Almenárez: PDP: A lightweight discovery protocol for local-scope interactions in wireless ad hoc networks. Computer Networks, 50(17) (2006) 3264-3283

5. M.J., Kim, M., Kumar, B.A., Shirazi: Service Discovery using Volunteer Nodes for Pervasive Environments. Proceedings of International Conference on Pervasive Services, (2005) 188-197 